\title{
CBRN Events and Crisis Communication: Analysis of Training Needs and Development of Curriculum for Communication Personnel
}

\author{
Saman Choudary ${ }^{*}$, Muhammad Usman Asghar ${ }^{2}$, Ahmed Gamal Ibrahim ${ }^{3}$ \\ ${ }^{1}$ Department of Communication, Pompeu Fabra University, Barcelona 08018, Spain \\ ${ }^{2}$ Center for International Peace and Stability, National University of Sciences and Technology, Islamabad 44000, Pakistan \\ ${ }^{3}$ International Master Courses in Protection Against CBRNe Events, University of Rome Tor Vergata, Rome 00133, Italy
}

Corresponding Author Email: saman.choudary01@estudiant.upf.edu

https://doi.org/10.18280/ijsse.110406

Received: 15 February 2021

Accepted: 20 July 2021

Keywords:
CBRN event, crisis communication,
communication personnel, training
curriculum

\begin{abstract}
Crisis communication is one of the key components of chemical, biological, radiological and nuclear (CBRN) events management and the successful management of a CBRN event depends on the execution of effective and timely crisis communication mechanisms, institutionalized in the response network. A CBRN event in form of an accident, incident or terrorism attack presents a unique environment and challenges for communication, which requires a specialized communication response, based on well-tailored education and training of the communication personnel. This calls for development of an objective and comprehensive approach towards crisis communication, indoctrinated and designed in a well-defined curriculum based on theoretical scholarship and operational training. Therefore, the aim of this study was to determine and address the needs for CBRN related crisis communication and to develop a CBRN training curriculum for communication personnel. This research was pursued by means of needs assessment method involving an online survey targeted towards specific set of stakeholders involved within the field of CBRN defense and crisis communication. Situational awareness, knowledge about risks, operational information, and training in terms of understanding the origin of CBRN events, chain of response network, message formulation, conducting interviews, and employment of communication techniques while reporting on specific CBRN scenarios have been identified as specific training needs. On the basis of the findings of the survey, the training curriculum has been developed and suggested, which will contribute to the sufficient communication training of communication personnel, leading to development of extensive communication competencies and skills in the field of CBRN defense, benefiting the nation-states.
\end{abstract}

\section{INTRODUCTION}

The world is suffering from the deadly-outbreak of COVID19 that evolved from an epidemic to a global pandemic in few months and swamped the world carrying catastrophic human, social, economic and psychological consequences [1]. The information environment on COVID-19 is constantly evolving, encompassing a surge of misinformation being characterized by inconsistent and unclear messages concerning risk levels and appropriate protection actions [2]. This has given rise to an "infodemic" symbolizing COVID-19 as the first pandemic of the social media age $[3,4]$. In the early phases of this global pandemic, inadequate communication and information mismanagement has played a key role in the spread of the disease and fueling public misperceptions and distrust further worsening the management and control of the epidemic [5]. In addition, the response organizations down played the urgency of the matter and no warnings were given to the public for crisis preparation and for taking informed precautionary measures resulting into mass contaminations and chaos [5].

The rapid inter-species transfer of (COVID-19) holds a strong relevance to chemical, biological, radiological and nuclear (CBRN) defense, being classified as biological event falling under the category of biological threats while having natural origin in form of infectious disease caused by Severe Acute Respiratory Syndrome CoronaVirus 2 (SARS-CoV-2). The COVID-19 pandemic has brought forward the centrality of effective crisis communication as a force for adequately dealing and managing a health emergency and shaping public perceptions based on factual and well-informed crisis communication. COVID-19 as a biological threat presents a seriously challenging situation, characterized by complexity and uncertainty, requiring a quick and timely response from world leaders, health organizations and mass media. The evolution of this crisis as biological emergency has demonstrated that states have to be prepared more prudently to address CBRN threats that could take the turn for worst in a matter of days.

As exhibited by COVID-19, the crisis communication capabilities cannot be ignored and demand adequate planning as an integral part of public health emergency preparedness and CBRN events. A timely and well-informed communication can have positive impact on public responses in terms of fostering appropriate behaviors and trust besides confidence building in state policies and response. However, the lack of resources, competences and cooperation in the response network regarding crisis communication are the main and common issues that are needed to be addressed on priority 
and to develop an effective crisis response system and the related communication strategy for events involving CBRN related materials [6].

In CBRN events, communication personnel are the vital resource personnel to act as first responders to deal and manage the emergency in terms of providing critical information input and updates to the public in early hours of the emergency. However, it is feared that these personnel are not well prepared to deal with such kind of emergencies specifically lacking the capability, knowledge and awareness to communicate efficiently, thereby requiring specialized efforts targeted towards crisis communication preparedness of this group. It is needed to ensure that these actors are well equipped and trained to deliver accurate information to the masses in order to curtail the panic and chaos owing to varying public risk perceptions and uncertainty involved relating to element of surprise, lethality, and unseen agents [7].

In this backdrop, an effort has been carried out to address this gap as CBRN crisis presents unique environment and challenges for communication, which requires a specialized communication response, backed by well-tailored education and training of communication personnel. This calls for development of a collaborative and comprehensive approach towards crisis communication designed in a robust curriculum based on theory and operational training that could be used to train communication personnel. However in practice, there is no evidence of such specialized curriculum that could be used to train communication personnel as literature is enriched with research studies addressing different aspects of crisis communication but lack in the field training based on a comprehensive curriculum providing benchmark guidelines for communication personnel [8]. Therefore, this paper is aimed at defining the needs for CBRN communication and development of a CBRN training curriculum for communication personnel as an educative approach for clarifying their role, and quality criteria related to communication during CBRN events. The final product in form of the curriculum will contribute towards capacity building of the commination personnel yielding communication preparedness in the field of CBRN defense.

\section{CBRN DEFENSE, COMMUNICATION CHALLENGES, AND NEED FOR CURRICULUM}

CBRN defense generally serves as protective umbrella that deals with the threats emanating from unconventional materials that include chemical, biological, radiological and nuclear materials and provides a comprehensive set of protective measures and mechanisms against these threats. In holistic terms, CBRN defense constitutes the range of CBRN incident response activities, governance of domestic agencies involved in CBRN incident response, and, the capability to deal with CBRN related threats [9]. The vast field of CBRN defense encompasses a diverse category of stakeholders, subject expertise, skills, procedures, and, equipment that are all mustered up together to address the CBRN threat. The CBRN defense can be viewed in form of a model involving a specialized context of CBRN activities, assessment of threat, building capabilities for prevention, robust preparedness efforts, alerting and early response, and, remediation through reconstruction and recovery [10]. Prevention, preparedness and response are the prime pillars of CBRN defense that constitute the entire spectrum of this particular sector of defense, dealing specifically with CBRN events having natural or inadvertent origin. The robustness of the CBRN defense can be seen in terms of increased prevention, improved preparedness and enhanced response capabilities for the objective of reinforcing resilience and mounting defenses against CBRN attacks. The prevention pillar deals with the crucial aspect of limiting the likelihood of CBRN incidents in form of restricting access to the CBRN materials and identifying probable threats, the preparedness pillar deals with having sufficient capabilities and resources to respond to a CBRN threat if it materializes itself and lastly the response pillar addresses the consequence management in case of occurrence of a CBRN incident with an aim of containment and minimizing the damage along with recovery efforts [9]. In the wake of the growing wave of terrorist events around the globe, the possibility of terroristic attacks materializing the usage of CBRN materials cannot be truly ruled out. The materialization of these attacks as a crisis presents worst case scenario involving highly complex environment requiring precise and timely information and communication among response organizations and to the public [11].

These scenarios involving a deadly combination of hazardous materials and their usage by the terrorist entities could result into colossal damage encompassing mass causalities, uncertainty and chaos among public groups yielding psychological turmoil and unexpected responses necessitating timely communication and communication actions [6]. In this context, crisis communication is the fundamental instrument to respond in such a challenging phase of CBRN terrorism crisis. Crisis communication in relation to CBRN terrorism has been lately studied in depth to analyze and address the communication challenges leaving room for further research in this area and proposing fundamental solution-oriented instruments to deal with these challenges and enhancing preparedness [11].

A review of the current prevailing instruments indicates that no concrete steps have been taken to address the problems faced by key communication personnel involved in the reporting of the information as communicators at the first level of response chain that presents an under researched area [12]. The communication personnel could be defined as the persons from news media entities involved in the direct reporting of the information at the location of the incident such as journalists and reporters [13]. Communication personnel have a generalized view of the crisis caused by different factors but lack in-depth awareness about the materials involved into a CBRN event such as hazardous materials (HAZMAT), \& CBRN agents, which could lead towards misinformation of critical facts and cause of panic and confusion in general masses. These actors fall short of competency, skills and resources in the response chain to communicate precisely and effectively with public as iterated by the study conducted by Ruggiero [6].

There is a need to ensure that communication personnel are clear about their roles and have adequate competency to conduct crisis communication in the wake of a CBRN terrorism crisis [14]. Thereby, crisis communication has been approached to enhance preparedness of response personnel for public communication within the different phases of crisis communication cycle involving: pre-crisis phase; initial phase; maintenance phase; resolution phase; and evaluation phase [15]. While each phase carries specific communication requirements for the aim of effectively managing the crisis. As a critical part of preparedness, crisis communication personnel 
have been taken as a specific target group that require adequate education in the form of a specialized training in order to play an active and informed role in the management of a CBRN event via public communication. The findings of this study will yield the development of a crucial instrument in the form of a training curriculum for this specific target group for enhancing their competency and skills.

\section{MATERIAL AND METHODS}

This study on CBRN terrorism and crisis communication has been carried out to develop a specialized curriculum for the specific critical actors i.e., communication personnel (journalists and reporters). The study has been conducted with an aim of answering following research questions:

RQ 1: Why communication personnel require a comprehensive curriculum to deal with any CBRN event?

RQ 2: What are the needs of the communication personnel for responding to a CBRN event?

RQ 3: What should be the basic structure and components of the training curriculum?

In order to seek answers to these questions, the specific method (Needs assessment) has been adopted and performed with the help of a survey based on questionnaires targeted towards a specific set of stakeholders involved within the field of CBRN defense and crisis communication [16]. The Needs assessment methodology follows six steps involving: stating the objectives; selecting target audience; collecting data; selecting audience sample; picking an instrument for data collection; analyzing data; and follow-up [17]. These steps have been undertaken in the investigative process and the results have been presented in form of figures and follow-up has been performed in form of decision making for designing a specialized training curriculum. Needs assessment helped in characterizing the perceived training needs and assisted in developing a well-informed curriculum corresponding to specialized learning needs. In this backdrop, the existing literature regarding crisis communication indicates that challenges and expert perceptions have been identified and explored by researchers which provide an indication about the specific areas to be addressed in the curriculum formulation [6]. The critical areas that have been taken into consideration involve critical resources, capabilities, and cooperation in communication preparedness and the time for provision of information in the evolution of the crisis. These areas along with identified needs have been integrated and addressed, while planning and developing the specialized curriculum that will contribute to the sufficient communication training of communication personnel, leading to development of extensive communication competencies and skills.

\subsection{Data collection}

The survey comprised of six specialized questionnaires having mainly closed-ended multiple choice questions along with few open-ended questions. In relation to open-ended questions, the respondents were requested to give input about the particular learning/training needs of the communication personnel involved in reporting of CBRN events and basic elements that are needed to be covered while training the communication personnel. For the purpose of enabling participants to respond to the questionnaire promptly, survey monkey was used as a survey tool for sending questionnaires and collecting responses

Six stakeholder groups were identified for the survey and a specialized questionnaire was sent to each specific stakeholder group. These stakeholders are as follows:

(1) Reporters and Journalists (Communication Personnel).

(2) Media Owners including owners of TV news channels, press, and journals.

(3) First Responders are the personnel that are responsible for responding to the incident in the first phase on emergency on the scene and carry out activities of (firefighting, medical assistance, cordoning, detection, warning, reconnaissance, sampling etc.).

(4) Incident Commanders are specific personnel who manage different aspects of the incident as in charge of the first response team and at the same time are a point of contact for primary real time communication and provision of information to the public communication officer.

(5) Academia and Academic Organizations

(6) CBRN Experts, Response Organizations and Policy Makers.

The sample of each stakeholder group personnel composed of specialists working in the specific sector and the selection was made in an organized manner based on a selection criterion (technical background \& experience), the recommendations and availability of the experts. Cumulatively 60 personnel received the questionnaires via an invitation email and web link whereas 47 submitted their responses in the form of filled questionnaires. Figure 1 demonstrates the occupation ratio of the questionnaire respondents.

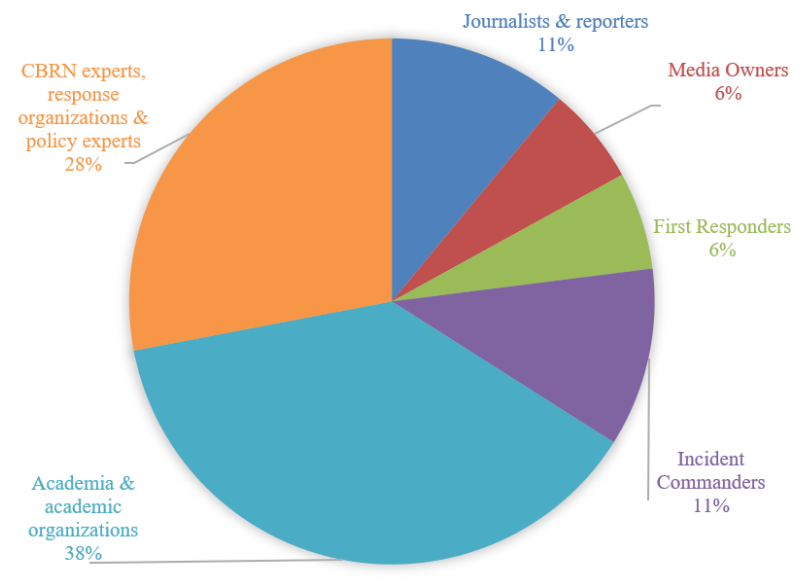

Figure 1. Occupation ratio of questionnaire respondents

Although, the number of respondents was not very high, their feedback provided valuable insights and answers to the RQ1, RQ2, and RQ3. The smaller number of respondents does highlight the need to further expand the sample size but given the time and pandemic related constraints, these limitations are mentioned as research limitations of the study. On the other side, this data set does provide an opportunity to have primary and initial baseline for the research.

\section{KEY FINDINGS AND ANALYSIS}

This section of the study explains the research findings and the interpretation of the results, while analyzing the various 
aspects of the responses as recorded during the inquiry from the target audience. The research findings are discussed based on the data collected from all six stakeholders (groups) on the need assessment for a specialized crisis communication curriculum. These findings demonstrate the state of awareness, preparedness, and competencies of the communication personnel, the main communication challenges, specific learning needs, components and structure of the curriculum for the effective development of the curriculum.

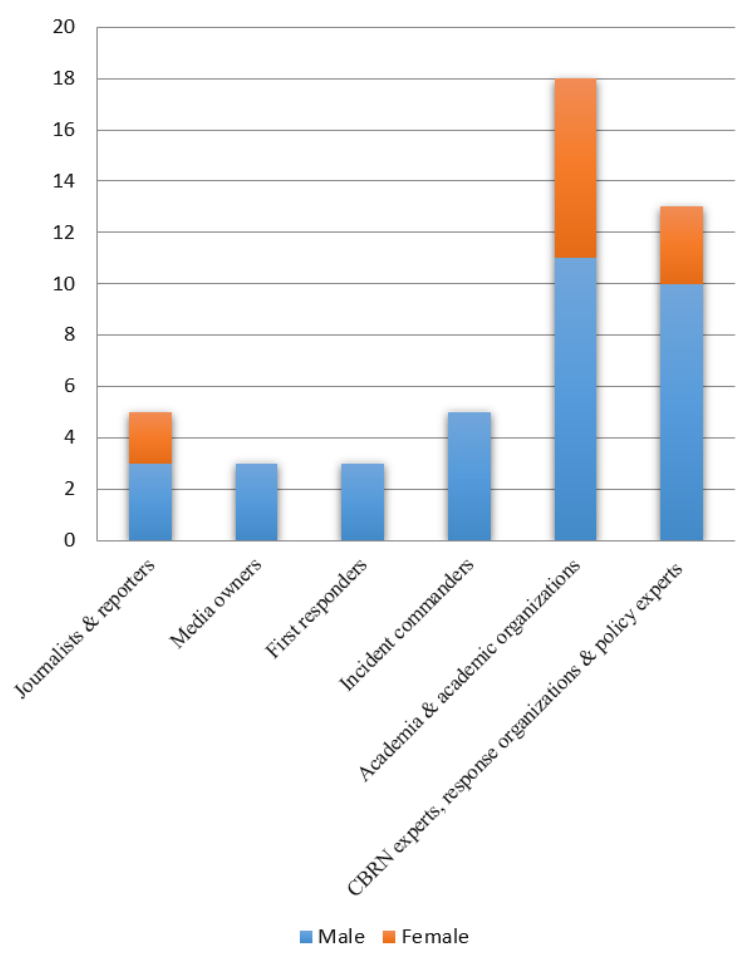

Figure 2. Category wise questionnaire responses

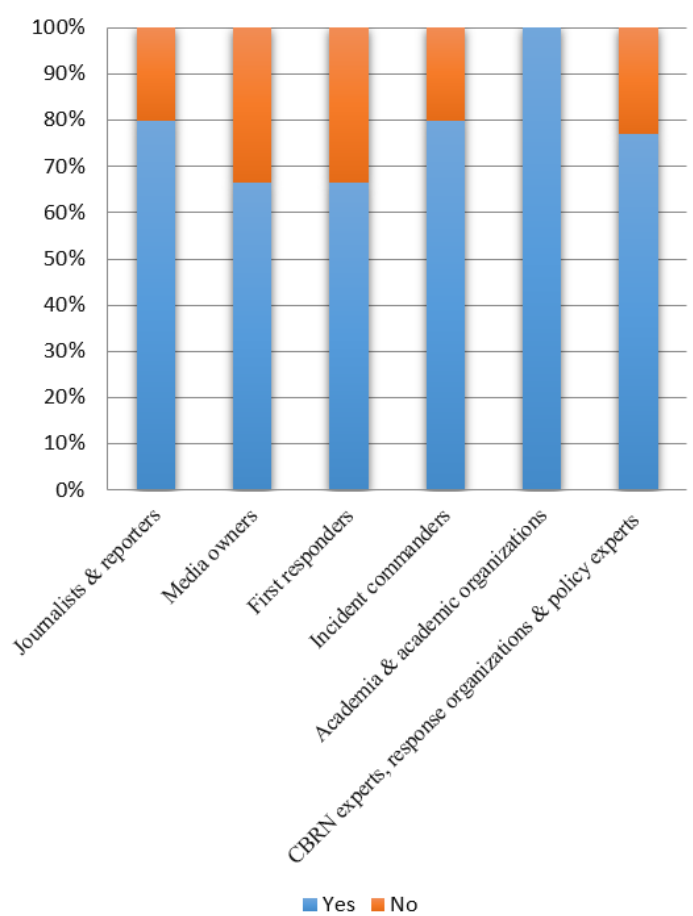

Figure 3. Need assessment for a specialized course on CBRN crisis communication
A total of 47 persons responded to the questionnaires and their breakup and groups are shown in Figure 2. The response is diversified representing the highest response rate from academia (18) and CBRN experts, organizations, and policy makers (13) respectively.

The first finding from the close-ended questions relates to key inquiry in form of the first research question of the study that attempts to establish the need for a specialized course for training and capacity building of the communication personnel involved in the crisis communication in the backdrop of a CBRN event. The results are presented in the graph (Figure 3). All the stakeholders responded positively to the need of for a specialized course for communication personnel that is $85 \%$ of respondents in total. However, only $15 \%$ of respondents collectively from all stakeholder groups answered that there is no need for the specialized course. This outcome indicates that this is a crucial area that has been neglected so far and is needed to be addressed on an immediate basis as agreed upon by all the concerned stakeholders involved within the response chain.

The second finding relates to the existence of the awareness and sufficient competencies of the communication personnel for responding to a CBRN event (see Figure 4). As per the feedback, 42 respondents suggested that there are not enough competencies and capabilities and the communication personnel lack in this area. On the contrary, only 5 persons responded that there are sufficient competencies to report on such events. These results provide direct insight into the real time competencies of concerned actors lacking basic knowledge and awareness of the HAZMAT and CBRN events further necessitating the development of specialized tools for addressing this gap.

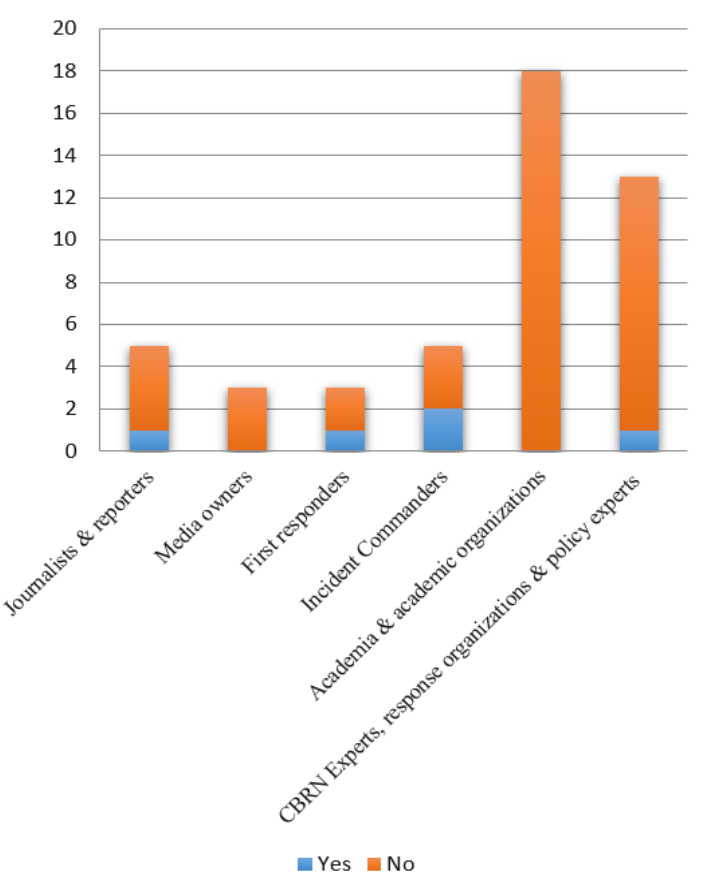

Figure 4. Level of satisfaction regarding CBRN crisis communication competencies

The third finding relates to the assessment of current capabilities of the communication personnel in terms of being taking part in a specialized training or course on the subject of communication and CBRN incidents as indicated by the graph given below (see Figure 5). The feedback indicated that 100\% 
of the respondents from the journalists and reporters group have not been a part of any specialized training or course relating to CBRN incidents, which symbolizes that their capabilities are under developed in this specific area of reporting.
Paricipated in any training/course on

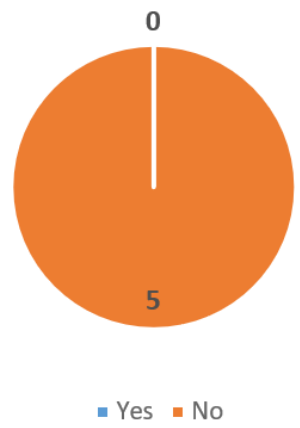

Figure 5. Feedback of journalists and reporters in specialized training or course on communication and CBRN incidents

The fourth finding relates to the resources at the disposal of communication personnel in order to be prepared for reporting on CBRN incidents as indicated by the graph (see Figure 6). The feedback indicated that $100 \%$ of the respondents from the journalists and reporters group do not have adequate resources in form of personnel protective equipment and training on their usage that is mandatory for reporting on a CBRN incident for ensuring safety.

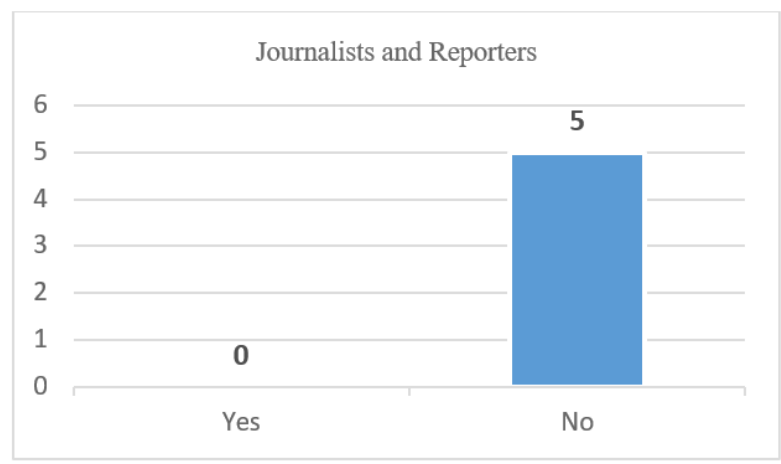

Figure 6. Feedback of journalists and reports on the availability of Personnel Protective Equipment (PPE) and PPE training

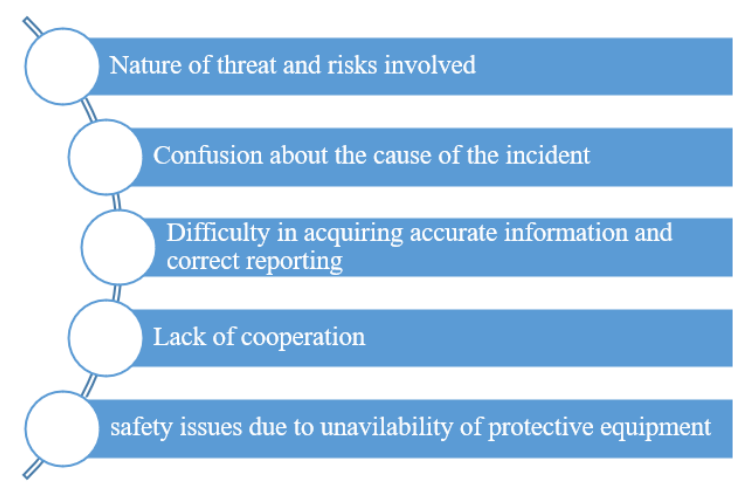

Figure 7. Feedback on challenges faced by reporters and journalists while covering $\mathrm{CBRN}$ events
The fifth finding relates to the challenges while communicating about the CBRN events. As suggested by the feedback of the first stakeholder group on this critical area, the responses are collectively represented in Figure 7. The outlined challenges attribute poor preparedness in terms of crisis communication that can evoke fear, confusion and panic in case of materialization of a CBRN threat into reality resulting into poor management and mishandling of the crisis.

The next finding provides an answer to the second key question of this research study that what are the needs of the communication personnel for responding to a CBRN event/emergency?

With regards to the needs of the communication personnel, the following needs have been identified as stated by the respondents of the questionnaires (see Figure 8).

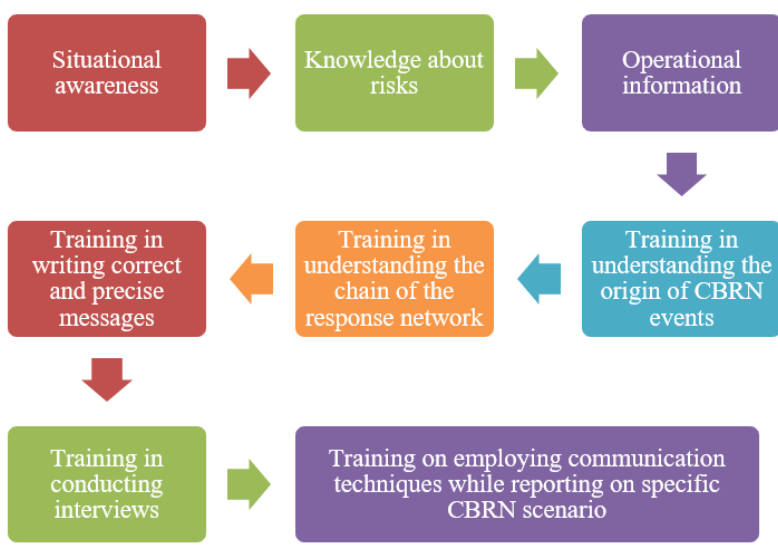

Figure 8. Needs Assessment

These needs relate to lack of theoretical background and operational experience for approaching the subject of CBRN events, requiring an extensive effort to build upon the knowledge base in terms of basic understanding of CBRN materials, background information, cognizance of potential risks and consequences, actors involved in generating the response, tactical information about crisis communication and messaging in relation to $\mathrm{CBRN}$ incidents, and practical training with the help of practical tools such as table top exercises. Based on the primary feedback, these needs have been prioritized and integrated into the suggested curriculum as primary building blocks.

Knowledge about CBRN events (Threats, effects and management)

Knowledge about origin of CBRN events and normal events

Specific aspects of communication to be taken in consideration under each CBRN scenario

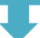

Knowledge of communication principles, reporting and messaging in CBRN crises

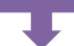

Knowledge of the basics of institutional communication and response chain

Practical training

Figure 9. Feedback on elements to be included into the curriculum 
Lastly, at the final stage of the analysis, the results have been drawn from the feedback for answering the third question of the study that is based on identifying the key elements of the training curriculum. The suggested components and elements have been presented in Figure 9, which are needed to be included in the curriculum.

With respect to RQ1, RQ2, and RQ3 the feedback from the concerned stakeholders has provided evidence of the real time competencies and capabilities of communication personnel while characterizing the perceived needs. The identified needs are consistent with the communication challenges highlighted in the previous studies on crisis communication and CBRN terrorism being conducted by Ruggiero in the year 2015 .

To move forward and to complement Ruggiero's effort the suggested curriculum as a communication preparedness instrument is presented in the proceeding section.

\section{PROPOSED CURRICULUM AND STRUCTURE}

Based on the outlined results of the survey the training curriculum has been proposed and designed that follows the foundational structure of the NATO's international curriculum for first responders [18]. The training curriculum is divided into six learning outcomes that are also referred to as modules. Each learning outcome can be built upon a number of learning objectives comprising of different components that support the overall outcome. Furthermore, several teaching points can be developed that provide the understanding to achieve the learning objectives. As per the NATO's approach, the rationale behind the specifically designed structure could be understood as if teaching points are understood; the learning objective is attained, leading to the achievement of the learning outcome. Given the limitation of space and words, only the learning outcomes have been presented here excluding the learning objectives and teaching points (see Figure 10)

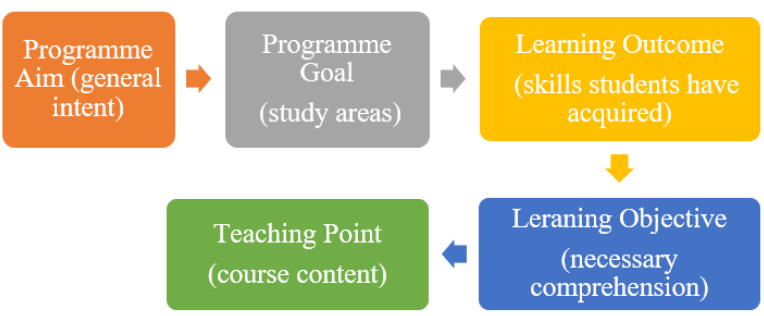

Figure 10. Structure of the training curriculum

The learning outcomes are developed while following a depth of knowledge approach moving from simpler concepts to complex thinking and learning process (see Figure 11).

Firstly, the programme aim and goal have been defined shaping a road map to the building blocks of the curriculum. At first step, the foundational background has been deliberated in order to develop the basic understanding of the learners for the evolution of this particular domain of defense (CBRN preparedness and defense) in the international security environment. At the second step, the fundamentals of CBRN threats, risks and scenarios have been placed to provide an overview of these threats and management components. At the third step, crisis communication has been introduced in relation to the management of CBRN events characterizing the application of basic concepts, principles, and techniques of emergency communication in all the phases of the crisis. At the fourth step, communication strategies for dealing with different CBRN scenarios have been placed as the communication needs differ for each specific scenario involving a specific CBRN agent. At the fifth step, reporting aspects in particular procedures and strategies have been focused on relating to information gathering, message formulation and communicating with different public groups. At the final step, table top exercises have been suggested for the practical application of emergency communication techniques and procedures by the learners in order to finalize and gauge the learning outcomes of the curriculum. It is proposed that the training instructors can tailor the specified learning outcomes of the curriculum to meet the specific needs of the audience.

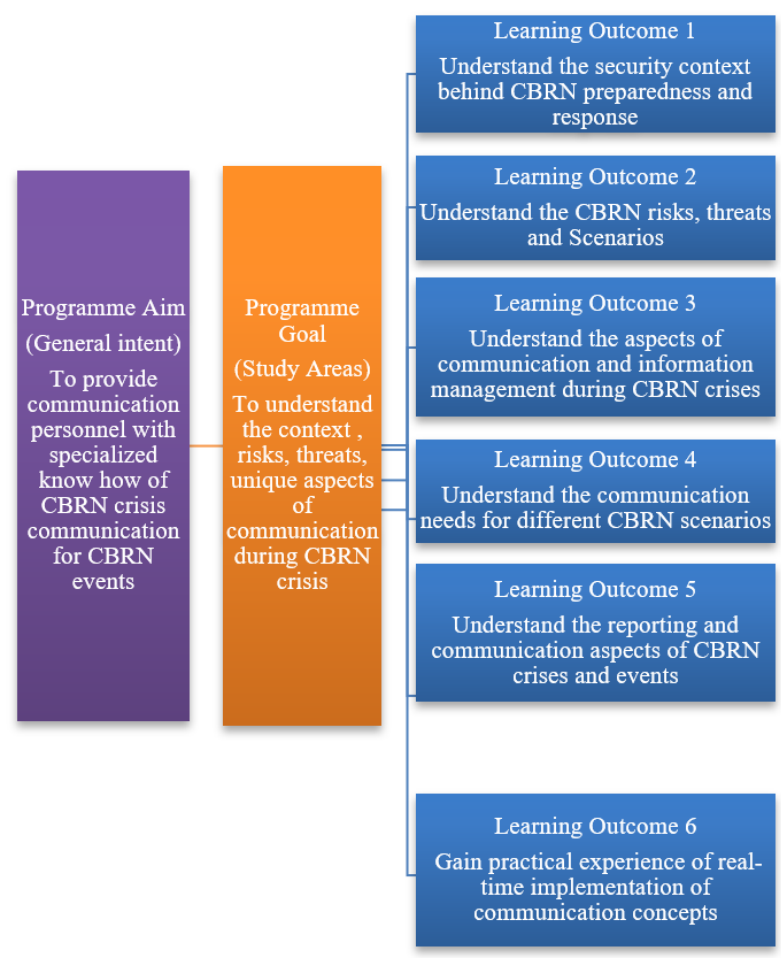

Figure 11. Designed Curriculum

\section{CONCLUSION}

The devastating impact of the COVID-19 pandemic as a biological event indicates the criticality of developing tools for improving crisis communication and preparedness of response organizations within the CBRN response network. The terrorism incidents caused by the potential usage of CBRN materials could make communication a very challenging task that requires specific educational and training programmes for enhancing knowledge, competency, and expertise of communication personnel. They need comprehensive knowledge of the communication fundamentals and appropriate communication strategies for responding to a crisis particularly linked to CBRN agents. This proposal for an innovative curriculum to prepare communication personnel to intervene quickly as well trained communicators in the crisis communication component of the overall crisis management process of CBRN emergencies will significantly prove to be an effective instrument for strengthening CBRN defense. The curriculum is based on academic research involving feedback 
from concerned stakeholders while containing theoretical and practical elements. This research study represents a commitment to international security in terms of a continuous effort for the development and consolidation of enabling tools and instruments within the realm of CBRN preparedness and response. To conclude, a special request has been made to policy making and defense sector for the practical materialization of the proposed instrument that will promote communication preparedness in turn warranting effective management of CBRN events.

\section{REFERENCES}

[1] Zhang, S., Zhaou, L., Lim, J. (2020). From networking to mitigation: the role of social media and analytics in combating the covid-19 pandemic. Information Systems Management, 37(4): 318-326. https://doi.org/10.1080/10580530.2020.1820635

[2] Krause, N.M., Freiling, I., Beets, B., Brossard, Dominique. (2020). Fact-checking as risk communication: The multi-layered risk of misinformation in times of COVID-19. Journal of Risk Research, 23(7-8): 1052-1059. https://doi.org/10.1080/13669877.2020.1756385

[3] Viswanath, K., Lee, E.W.J., Pinnamaneni, R. (2020). We need the lens of equity in COVID-19 communication. Health Communication, 35(14): 1743-1746. https://doi.org/10.1080/10410236.2020.1837445

[4] World Health Organization. (2020). Novel Coronavirus (2019-nCoV) Situation Report-13. https://www.who.int/docs/defaultsource/coronaviruse/situation-reports/20200202-sitrep13-ncov-v3.pdf. accessed on Dec. 7, 2020.

[5] Fu, K., Zhu, Y. (2020). Did the world overlook the media's early warning of COVID-19? Journal of Risk Research, 23(7-8):

https://doi.org/10.1080/13669877.2020.1756380

[6] Ruggiero, A., Vos, M. (2015). Communication challenges in CBRN terrorism crises: Expert perceptions. Journal of Contingencies and Crisis Management, 23(3): 138-148.

[7] Covello, V., Peters, R., Wojtecki, J., Hyde, R. (2001). Risk communication, the west nile virus epidemic, and bioterrorism: responding to the communication challenges posed by the intentional or unintentional release of a pathogen in an urban setting. Journal of Urban Health, 78(2): 382-391.

[8] Ruggiero, A., Vos, M. (2013). Terrorism communication: characteristics and emerging perspectives in the scientific literature 2002-2011. Journal of Contingencies and Crisis Management, 21(3): 153-166.

[9] The National Institute for Defense Studies. (2015). East Asian Strategic Review. http://www.nids.mod.go.jp/english/publication/eastasian/e2015.html, accessed on Nov. 29, 2020.

[10] Healy, M.J.F., Weston, K., Romilly, M., Arbuthnot, K. (2009). A model to support CBRN defence. Defense \& Security Analysis, 25(2): 119-135. http://dx.doi.org/10.1080/14751790902985157

[11] Ruggerio, A. (2017). Crisis communication and terrorism: Mapping challenges and co-creating solutions. Ph.D. dissertation. Department of Communication, University of Jyväskylä, Jyväskylä, Finland.

[12] Gloria, A. (2018). International training curriculum for advisors in emergencies and CBRNE events management. In: Malizia A., D’Arienzo, M (eds) Enhancing CBRNE safety \& security: proceedings of the SICC 2017 conference, https://doi.org/10.1007/978-3319-91791-7 48

[13] Ruggiero, A., Vos, M., Palttala, P. (2015). The CBRN communication scorecard. In: Schmidt. S., Vos, M. (eds) Behaviour and communication in CBRN crisis. Findings and recommendations in case of chemical, biological, radiological, and nuclear attacks on society. Pabst Science Publishers, Lengerich, 106-139.

[14] Mykkänen, M., Vos, M. (2014). Clarifying the role of communication experts in CBRN terrorism crises. Report project CATO. University of Jyväskylä, Finland.

[15] Centers for Disease Control and Prevention (CDC). (2014). Crisis and emergency risk communication (manual).

https://www.nphic.org/Content/ProfDev/Documents/cer c_2014edition.pdf, accessed on Nov. 29, 2020.

[16] Grant, J. (2002). Learning needs assessment: assessing the need. BMJ (Clinical research ed.), 324: 156-159. https://doi.org/10.1136/bmj.324.7330.156

[17] McCawley, P.F., (2009). Methods for conducting an educational needs assessment. University of Idaho. https://www.extension.uidaho.edu/publishing/pdf/BUL/ BUL0870.pdf. accessed on Dec. 2, 2020.

[18] NATO Civil Emergency Planning Civil Protection Group. (2013). Project on minimum standards and nonbinding guidelines for first responders regarding planning, training, procedure and equipment for chemical, biological, radiological and nuclear (CBRN) incidents: the international CBRN training curriculum. https://www.nato.int/docu/cep/cep-cbrn-training-e.pdf. 